\title{
La Validation des Acquis de l'Expérience De la démarche personnelle à la réalisation encadrée : Une expérience à l'université Bordeaux 1
}

\author{
Isabelle Sand ${ }^{1}$, Geneviève Duchamp ${ }^{2}$
}

isabelle.sand@u-bordeaux1.fr, geneviève.duchamp@ims-bordeaux.fr

Université Bordeaux1

${ }^{1}$ Service Commun de la Formation Continue et de l'Apprentissage, ${ }^{2}$ UFR de Physique 351 cours de la Libération 33405 TALENCE - Cedex

\begin{abstract}
RESUME : Le dispositif de Validation des Acquis de l'Expérience (VAE) a été mis en place par l'université Bordeaux 1 en application de la loi de modernisation sociale du 17 janvier 2002. Après un rappel du cadre législatif et une présentation du contexte local, l'article met l'accent sur le rôle des acteurs impliqués dans une succession de rencontres-étapes avec le candidat qui donnent à la procédure VAE sa dimension pédagogique. Il met en lumière l'approche de Bordeaux 1 sur les aspects de la démarche qui relèvent du domaine de décision des universités. Il présente aussi, à partir des expérimentations en cours, les effets de ce dispositif sur les modalités pédagogiques de formation en vigueur à l'université. Enfin, des éléments de bilan et les enjeux pour l'avenir du dispositif viennent compléter cette présentation.
\end{abstract}

Mots clés : dispositif pédagogique, formation continue, public adulte, validation des acquis, expérience professionnelle, transfert de savoir-faire, retour d'expérience.

\section{INTRODUCTION}

Dans son rapport « La formation professionnelle - Diagnostics, défis et enjeux » publié en 1999, Nicole PERY, Secrétaire d'Etat au droit des femmes et à la formation professionnelle formule l'enjeu de la validation des acquis de l'expérience en ces termes :

«Comment vont s'adapter, face aux mutations qui s'accélèrent, les $40 \%$ d'hommes et de femmes de la population active actuelle ayant une formation initiale inférieure au C.A.P. et qui sont souvent exclus de fait de la formation continue? Beaucoup heureusement ont acquis des savoir-faire par leur expérience professionnelle. Comment les valider afin qu'ils fassent l'objet d'une reconnaissance plus large à travers des repères élaborés collectivement ? »

De même le technicien supérieur, le manager de $1^{\text {er }}$ niveau ou le cadre confirmé peinent à faire reconnaître leur niveau lorsqu'ils changent d'entreprise ou postulent à un poste supérieur s'ils n'ont pas obtenu, lors de leur formation initiale, un $\mathrm{Bac}+3$ ou un $\mathrm{Bac}+5$.

La Validation des Acquis de l'Expérience (VAE), instituée par la loi de modernisation sociale du 17 janvier 2002 a ainsi l'ambition de donner plus de fluidité au marché de l'emploi en permettant aux actifs de faire reconnaître les compétences acquises en cours de carrière par l'obtention d'un diplôme ou titre professionnel.

A l'université Bordeaux1, entre 2004 et 2008, 54 candidats ont obtenu une validation partielle ou totale dans le cadre de cette procédure pour une centaine de dossiers suivis par an.

Après une brève présentation du cadre légal dans lequel s'inscrit la VAE, nous présenterons le contexte dans lequel la procédure a été mise en place à Bor- deaux1, les acteurs du dispositif et le déroulement de la procédure. Nous détaillerons les points clés de la démarche ainsi que l'impact de ce nouveau dispositif sur les pratiques pédagogiques des filières. Enfin, un premier bilan permettra de proposer quelques pistes pour le développement futur de la VAE.

\section{CONTEXTE}

\subsection{Cadre législatif}

La loi de modernisation sociale du 17 janvier 2002 a modifié à la fois le Code de l'Education et le Code du Travail et stipule «Toute personne engagée dans la vie active est en droit de faire valider les acquis de l'expérience, notamment professionnelle, en vue de l'acquisition d'un diplôme (...). »

"Les diplômes (...) sont obtenus par les voies scolaire et universitaire, par l'apprentissage, par la formation continue ou, en tout ou partie, par la validation des acquis de l'expérience.

La validation des acquis produit les mêmes effets que les autres modes de contrôle des connaissances et aptitudes.

Peuvent être prises en compte, au titre de la validation, l'ensemble des compétences professionnelles acquises dans l'exercice d'une activité salariée, non salariée ou bénévole, en rapport direct avec le contenu du diplôme(...). La durée minimale d'activité requise ne peut être inférieure à trois ans. »

Le décret no 2002-590 du 24 avril 2002 (...) relatif à la validation des acquis de l'expérience par les établissements d'enseignement supérieur précise :

«Peuvent donner lieu à validation, les acquis de l'expérience correspondant à l'exercice, continu ou non, pendant une durée cumulée d'au moins trois ans, d'activités salariées, non salariées ou bénévoles. Ces acquis doivent justifier en tout ou partie des connais- 
sances et des aptitudes exigées pour l'obtention du diplôme postulé. »

\subsection{Contexte local}

A Bordeaux 1, la pratique de la Validation des Acquis Professionnels "diplômante " avait été initiée dans le cadre du dispositif "VAP93 » mis en œuvre par une loi de 1992 et un décret de 1993. Ce dispositif prenait en compte les activités professionnelles d'une expérience d'au moins cinq années et permettait de délivrer toutes les unités d'enseignement d'un diplôme moins une.

La procédure VAE adoptée par le Conseil d'Administration. de l'université en 2004 reprend les modalités spécifiques à la mise en œuvre de la VAP93 à Bordeaux 1, qui constituent des points forts de la procédure actuelle :

- jury unique transdisciplinaire (physique, mathinfo, chimie, Sc. Biologiques, Sc. De la Terre et de la Mer)

- pilotage par le service de formation continue

- implication des responsables pédagogiques dès le début de la démarche.

Deux éléments nouveaux sont à souligner :

- la mise en place d'un accompagnement du candidat tout au long de la procédure

- le développement de pratiques de validation par le jury VAE visant à réellement prendre en compte la spécificité de ce public et à se démarquer d'une évaluation scolaire des connaissances et capacités des candidats.

Ces points forts seront développés ci-après.

Par ailleurs, il est à noter que les 4 universités de Bordeaux se sont concertées pour élaborer des documents communs, une même procédure VAE et des tarifs analogues. Le jury est propre à chaque université.

\section{DEMARCHE ADOPTEE, ELABORATION DE LA PROCEDURE VAE ET MISE EN EUVRE}

\subsection{Les acteurs « principaux " et " secondaires »}

La démarche VAE repose sur une suite de rencontres avec les acteurs clés du dispositif, qui sont des jalons, tant pour le candidat que pour l'université. Pour le candidat, il s'agit de prendre en compte les attentes spécifiques de l'université, d'une part, donner à connaître son histoire professionnelle, ses atouts, ses projets d'autre part. Pour l'université, ces rencontres-étapes permettent de découvrir le monde professionnel du candidat et de faire connaissance avec un individu et sa manière particulière d'exercer, sa professionnalité.

Les acteurs principaux sont, par ordre d'intervention dans le déroulement de la procédure VAE, la chargée de VAE et son assistante au sein du Service Commun de la Formation Continue et de l'Apprentissage (SCFCA), le responsable pédagogique du diplôme visé, l'accompagnateur méthodologique, les rapporteurs chargés d'évaluer les candidatures pour le compte du jury VAE, le Président et les membres du jury VAE enfin.

Dans l'étape de recevabilité, le candidat est accueilli par le service de la formation continue et de l'apprentissage (SCFCA), rôdé à l'information de professionnels qui connaissent peu le monde universitaire.

Si le projet du candidat est juridiquement éligible, un entretien d'orientation et de conseil est organisé avec le responsable pédagogique du diplôme et la chargée de VAE de l'université. Fonctionner en binôme est important. Cela permet une co-construction avec le candidat d'un parcours VAE tenant compte du référentiel du diplôme, des modalités pédagogiques d’un éventuel apport de connaissances complémentaires, de la bonne intégration de la démarche VAE dans le contexte professionnel et familial ou personnel du candidat, des contraintes financières enfin.

La coopération, dès le début de la procédure, de la filière pédagogique et du service dédié aux personnes engagées dans la vie active est un gage de cohérence et de cohésion pour l’université.

Ensuite, l'accompagnateur méthodologique prend le relais. Son rôle ? Aider le candidat à mettre en valeur son expérience professionnelle, ses connaissances, savoir-faire et aptitudes dans la rédaction de son dossier de VAE. Il est difficile d'écrire sur soi-même et d'avoir un regard objectif sur ses propres écrits. L'accompagnateur fournit ce regard distancié, aide à dégager les aspects essentiels de l'activité que l'on ne voit plus lorsqu'on a " la tête dans le guidon », encourage le candidat à se raconter, le remotive si nécessaire... car l'exercice est exigeant et parfois fastidieux!

L'accompagnement méthodologique contribue nettement à la qualité des dossiers présentés en jury. Les candidats accompagnés à cette étape vont tous jusqu'au bout de la démarche et se présentent au jury VAE.

Vient ensuite l'étape de l'évaluation. 2 rapporteurs, experts du domaine de compétences du diplôme visé sont chargés par le président de jury d'évaluer les connaissances et compétences du candidat. 2 regards complémentaires : celui d'un enseignant de la filière et celui d'un professionnel extérieur à l'université. Ces rapporteurs, après avoir lu le dossier du candidat, s’entretiennent séparément avec lui.

La dernière rencontre est celle entre le jury VAE et le candidat. Le jury entend le responsable pédagogique et les 2 rapporteurs, s'entretient avec le candidat, puis délibère. 
Au final, une quinzaine d'acteurs auront rencontré le candidat avant qu'un diplôme lui soit délivré dans sa totalité ou pour partie. Celui-ci aura côtoyé l'université au cours de 7 ou 8 entretiens.

Ainsi, la VAE n'est pas une procédure administrative ; elle n'est pas non plus la remise d'un diplôme par l'université à un inconnu.

Cet accompagnement « de bout en bout » permet seul le rapprochement techniquement difficile entre un profil de compétences individuel construit dans le monde professionnel et un référentiel de diplôme adossé à un parcours universitaire.

$\mathrm{Au}$ titre des acteurs secondaires, on mentionnera l'entreprise employeur du candidat et les organismes de financement de la formation professionnelle, qui, lorsqu'ils prennent en charge le coût de la VAE, facilitent l'engagement du candidat dans la démarche et lui permettent de l'accomplir dans de bonnes conditions.

\subsection{La procédure}

1. Sur demande du candidat, envoi d'un prédossier.

Objectif du pré-dossier : Etude de la recevabilité juridique et pédagogique de la demande.

2. Dépôt du pré-dossier renseigné par le candidat auprès du Service Commun de la Formation Continue et de l'Apprentissage de l'université Bordeaux 1 et règlement des frais d'étude du pré-dossier.

3. Entretien d'orientation et de conseil avec le responsable pédagogique du diplôme et la chargée de VAE de l'université (sauf candidature juridiquement non recevable).

4. Si la candidature est recevable, envoi de la convention VAE.

5. Signature de la convention par le candidat - et le financeur le cas échéant -, et règlement de $30 \%$ des frais de VAE.

6. Envoi du dossier VAE. Constitution du dossier par le candidat, seul ou accompagné.

7. Réception du dossier renseigné et finalisé, accompagné du paiement du solde des frais de VAE et des droits d'inscription au diplôme.

8. Inscription du candidat à l’université.

9. Examen du dossier par 2 rapporteurs nommés par le Président du jury VAE (un professionnel du domaine et un enseignant de la filière).
Puis entretien de chaque rapporteur avec le candidat et rédaction d'un rapport au jury VAE.

10. Examen de la candidature par le jury VAE de l'université Bordeaux 1 composé d'un représentant de chaque composante, du directeur de l'IUT, du directeur du SCFCA et présidé par le vice-président du CEVU. Le responsable pédagogique et les deux rapporteurs concernés siègent avec le jury.

Entretien avec le candidat.

Décision du jury VAE sur la validation demandée.

\subsection{Les points clés}

- L'entretien tripartite d'orientation et de conseil en début de procédure,

- L’accompagnement méthodologique,

- Le jury unique et transdisciplinaire,

- La participation des professionnels au jury VAE,

- Le pilotage par le service de formation continue,

- La rédaction d'une documentation destinée à chacun des acteurs.

\subsection{Les variantes : VAE totale ou partielle}

Actuellement, une majorité des candidatures présentées en jury VAE débouchent sur une validation totale. Or, les diplômes universitaires ouvrant sur un vaste panel d'emplois, sanctionnent une diversité de capacités qui ne sont pas toujours réunies dans le parcours d'un candidat. En toute logique, il devrait y avoir de nombreuses VAE partielles. Ce n'est pas le cas et cela explique aussi le nombre somme toute assez faible des candidatures traitées par le jury VAE.

On touche ici à une des limites de la mise en œuvre de la VAE à l'université. Souvent, les candidats ne peuvent obtenir un congé de formation pour venir suivre un complément de formation. Dès lors, si un diagnostic de validation partielle est établi au départ de la procédure, ils préfèrent renoncer à leur projet.

Or, l'enjeu du développement quantitatif de la VAE à l'université - tant demandé par les instances gouvernementales - nous semble résider dans la combinaison de VAE partielles et de parcours de formation individualisés.

\section{EXPERIENCE ET PREMIER BILAN}

\subsection{Expérience(s) d'application :}

Afin d'élargir l'accès à la VAE, l'université Bordeaux1 expérimente de nouvelles modalités pédagogiques de 
formation destinées à venir en complément d'une démarche de VAE.

La $1^{\text {ère }}$ permet de répondre à une insuffisance de culture technique, technologique et/ou règlementaire. Un programme de lectures sera donné au candidat et le rapporteur de la filière interrogera le candidat lors de l'entretien sur ce qu'il a retenu de ses lectures.

Exemple : à un candidat visant un diplôme en maintenance aéronautique et provenant du monde militaire, on recommandera de se mettre à jour sur la réglementation de l'aéronautique civile, connaissance clé de l'ensemble des diplômes de Bordeaux1 dans ce champ.

La $2^{\text {ème }}$ consiste à proposer au candidat de rédiger une synthèse bibliographique ou de mener un projet sur un thème qui est hors du champ de son expérience, également à partir de lectures personnelles, mais avec quelques entretiens de cadrage avec un enseignantchercheur. La synthèse ou le rapport sera annexé au dossier VAE.

Exemple : deux candidats au Master EEA, parcours Achat Industriel de Composants Electroniques se sont vu proposer une conduite de projet encadrée par un enseignant sur un thème faisant appel à leurs connaissances professionnelles en vue de mieux répondre au référentiel du diplôme.

La $3^{\text {ème }}$ est une auto-formation encadrée. Un programme individualisé d'auto-formation est établi en concertation entre le candidat et le responsable pédagogique du diplôme. Un encadrement pédagogique individualisé à distance et des entretiens d'étape sont prévus. Une évaluation écrite est organisée. Cette dernière formule s'inscrit dans le cadre d'une convention de formation.

Exemple : un candidat à l'obtention du Master Chimie suit actuellement un programme d'auto-formation encadrée visant à approfondir les aspects théoriques de certaines des compétences qu'il exerce professionnellement.

Des parcours individualisés de formation en présentiel avec les étudiants sont également proposés lorsque le candidat réside à proximité de l'université. Ces parcours peuvent porter sur des unités d'enseignement mais aussi sur des parties d'UEs, à partir d'une analyse précise des lacunes du candidat.

Dans tous les cas, ces expérimentations ont permis la délivrance totale du diplôme. Mais elles sont encore trop peu nombreuses pour en faire un premier bilan. Elles demandent flexibilité et disponibilité de la part des équipes pédagogiques, ce qui est possible pour un candidat de temps en temps, mais poserait sûrement des problèmes en cas de développement quantitatif de ces cas particuliers.

Aussi, l'université se tourne-t-elle à présent vers le développement de formations à distance qui se combi- nent idéalement avec une démarche de VAE. Un candidat a ainsi pu valider un Master MIAGE (Méthodes Informatique Appliquées à la Gestion des Entreprises) en suivant 6 UEs dans le cadre de la formation à distance après une VAE partielle.

\subsection{Retour d'expérience des équipes pédagogiques.}

Pour les équipes pédagogiques impliquées, les retours d'expérience font apparaître les principaux points suivants.

Tout d'abord, lorsque l'équipe est sollicitée pour la première fois pour une démarche VAE, la compréhension du dispositif et de sa mise en œuvre est une première étape qui prend du temps, suscite des questions et nécessite souvent de nombreux échanges notamment avec le SCFCA. De plus, pour les VAE suivantes, l'investissement humain pour l'accompagnement du candidat reste conséquent.

Par ailleurs, en l'absence d'évaluation chiffrée (pas de notes), la question se pose d'établir une forme de «compensation » entre les points forts et les points faibles identifiés dans les connaissances et compétences des candidats. Ceci peut entraîner une certaine différence de traitement entre formation initiale et VAE pour l'attribution d'un même diplôme, et nécessite une attention toute particulière de l'équipe pédagogique.

Enfin, s’agissant véritablement du développement de nouvelles modalités pédagogiques de formation, la mise en œuvre du dispositif et ses évolutions sont des sources d'amélioration des pratiques pédagogique pour l'ensemble de la filière (autoformation encadrée, projets de synthèse, équilibre formation présentielle / à distance...).

\subsection{Bilan « chiffré " :}

\subsubsection{Flux - taux de réussite}

\begin{tabular}{|l|c|c|c|c|c|}
\hline & $\mathbf{2 0 0 4}$ & $\mathbf{2 0 0 5}$ & $\mathbf{2 0 0 6}$ & $\mathbf{2 0 0 7}$ & $\mathbf{2 0 0 8}$ \\
\hline $\begin{array}{l}\text { Démarches } \\
\text { initiées }\end{array}$ & 109 & 100 & 113 & 113 & 118 \\
\hline $\begin{array}{l}\text { VAE } \\
\text { accordées }\end{array}$ & 5 & 9 & 14 & 14 & 14 \\
\hline $\begin{array}{l}\text { Dont VAE } \\
\text { totales }\end{array}$ & 4 & 2 & 11 & 13 & 14 \\
\hline $\begin{array}{l}\text { VAE refu- } \\
\text { sées }\end{array}$ & 0 & 0 & 2 & 0 & 0 \\
\hline
\end{tabular}

Répartition LMD depuis 2006 :

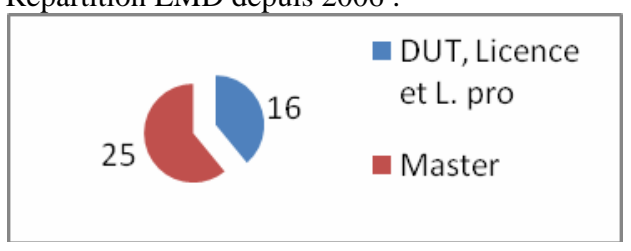

A titre d'exemple, en 2008, les 14 candidats qui se sont présentés au jury ont tous obtenu une validation totale. Ils représentaient $12 \%$ du nombre de candidatures reçues et $20 \%$ des dossiers recevables. $71 \%$ ont bénéficié d'un accompagnement méthodologique à la rédaction 
du dossier.

43\% des candidats ont bénéficié d'un financement par leur employeur; les autres ont bénéficié d'un financement partiel ou total de la part d'autres organismes ou ont financé personnellement leur démarche.

Enfin, pour ce qui concerne la composante UFR de Physique, sur la période considérée de 2005 à 2008, il y a eu 18 VAE menées à leur terme principalement dans les départements de mécanique et d'EEA. Les diplômes validés se répartissent de la façon suivante : 14 masters et 4 licences pro.

Bordeaux1 reste un " petit » valideur à l'échelle nationale, placé en $71^{\mathrm{e}}$ position sur 81 universités pour le nombre de dossiers présentés en jury, mais en $5^{\mathrm{e}}$ position pour la proportion de validations totales dans les décisions favorables du jury. Il se situe de ce fait à la $57^{\mathrm{e}}$ place en nombre de diplômes délivrés (données 2007).

Les résultats sont analogues si l'on retient un panel de 10 universités scientifiques (Bordeaux1,GrenobleI, LilleI, LyonI, MontpellierII, NancyI, RennesI, ToulouseIII, ParisVI, ParisXI) : Bordeaux1 se situe en dernière position pour le nombre de dossiers présentés, mais en $2^{\mathrm{e}}$ position pour la proportion de validations totales dans les décisions favorables du jury.

\subsubsection{Impact sur la carrière professionnelle}

Nous ne disposons pas réellement d'éléments factuels, hormis des remerciements appuyés dans la plupart des cas, sur le ressenti des candidats quant à la procédure mise en place et l'ensemble de la démarche. Cependant nous demandons à chaque candidat ayant obtenu une validation de nous tenir informés dans la mesure du possible de leur évolution professionnelle. Ainsi chaque année, les vœux du nouvel an, sont un prétexte à procéder à une rapide enquête de suivi. Le tableau cidessous reprend les éléments synthétisés sur la période 2005 - 2008 (hors candidats IUT).

\begin{tabular}{|l|l|l|l|}
\multicolumn{4}{|c}{ Evolution de la carrière professionnelle } \\
\hline $\begin{array}{l}\text { Taux de } \\
\text { réponse }\end{array}$ & $\begin{array}{l}\text { Evolu- } \\
\text { tion } \\
\text { positive }\end{array}$ & $\begin{array}{l}\text { Dont pro- } \\
\text { motion }\end{array}$ & $\begin{array}{l}\text { Dont changement } \\
\text { employeur ou retour à } \\
\text { l'emploi }\end{array}$ \\
\hline $62 \%$ & $83,3 \%$ & $44,4 \%$ & $27,7 \%$ \\
\hline
\end{tabular}

\subsubsection{Coût « direct » et « indirect »}

Le coût facturé est inférieur au coût réel de la VAE pour l'université. S'il permet de financer les dépenses directes liées à la rémunération des acteurs (hors SCFCA), il ne permet pas de couvrir intégralement le coût de pilotage du dispositif par le SCFCA. A titre d'exemple, le coût global annuel de la VAE pour l'université Bordeaux 1(hors IUT) est estimé à 37000 euros (coût total rémunération du personnel + quote part frais de fonctionnement du service hors locaux et charges correspondantes). Les recettes directes sont en moyenne de 9500 euros. Le coût de revient par candi- dat présenté en jury s’établit ainsi à un peu moins de $2000 €$.

Il est à noter que Bordeaux 1 propose une remise sur les frais de VAE aux candidats qui ne bénéficient d'aucune prise en charge financière.

\subsection{Difficultés rencontrées}

Le financement de l'activité VAE, laquelle ne bénéficie d'aucune subvention, reste déficitaire à Bordeaux 1. Des économies d'échelle n’ont pu être réalisées à ce jour pour les raisons suivantes :

- Accompagnement collectif peu adapté car les candidats sont dispersés sur le territoire national,

- Information des candidats chronophage du fait de la complexité des dispositifs de financement et de référentiels de diplômes trop succincts et peu visibles,

- Temps d'animation par le SCFCA qui reste important car, les acteurs sont encore souvent de nouveaux acteurs, compte tenu du nombre de diplômes proposés par l'université et donc du nombre de filières et rapporteurs potentiellement impliqués.

Lorsqu'un diplôme est demandé pour la $1^{\text {ère }}$ fois, l'appropriation du dispositif par l'équipe pédagogique de formation et par les rapporteurs demande de la part du pilote du dispositif une disponibilité certaine.

S'y ajoutent la formation des accompagnateurs méthodologiques, la rédaction de documents d'accompagnement des acteurs, la représentation de l'université dans les différentes instances publiques de gouvernance de la VAE.

\subsection{Points forts}

Les points forts que nous soulignons ici sont uniquement ceux liés à des choix de l'université Bordeaux1 ; ils ne se rencontrent pas dans toutes les universités.

Le pilotage par le service commun de la formation continue et de l'apprentissage facilite l'adoption d'une approche spécifique du public VAE par rapport au public étudiant. Il garantit la prise en compte du contexte professionnel et familial du candidat dans la démarche VAE. Il donne au candidat un référent commun à toutes les étapes de la démarche. Le chargé de VAE coordonne l'ensemble des acteurs et assure, de même que le jury unique, une homogénéité de traitement de toutes les candidatures.

L'entretien tripartite d'orientation et de conseil en début de procédure (responsable pédagogique, chargée de VAE, candidat) est un facteur clé de réussite de la démarche. Il permet d'évaluer la pertinence du projet et sa faisabilité à partir d'un diagnostic co-élaboré et, par conséquent, partagé.

Il permet d'anticiper les difficultés éventuelles et d'élaborer une réponse adéquate. Il donne au candidat 
les moyens de décider ensuite de poursuivre ou non sa démarche VAE avec un bon niveau d'information.

Le jury unique et transdisciplinaire pour tous les diplômes de l'université a permis d'élaborer des pratiques concertées en matière d'évaluation des candidatures. Celles-ci n'offrent en effet jamais un recouvrement complet avec le référentiel du diplôme. Le rapprochement des compétences du candidat de celles visées par le diplôme repose sur un savoir-faire que le jury a acquis progressivement. Par ailleurs, le jury unique contribue à homogénéiser les critères d'évaluation dans toutes les composantes de l'université.

\section{CONCLUSION}

L'université Bordeaux1 s'est impliquée dans la mise en œuvre du dispositif de VAE institué par le législateur en 2002, en identifiant des acteurs et un mode de pilotage qui lui valent un retour très positif de la part des candidats qui accomplissent l'ensemble de la démarche.

Après les premières années de mise en œuvre du dispositif, l'université vise dorénavant le développement de modalités pédagogiques de formation permettant aux professionnels d'accéder à des parcours de formation combinant VAE partielle, formation et poursuite de l'activité professionnelle.

La réorganisation depuis 2 ans de parcours présentiels en formations en alternance, qui concerne aujourd'hui 17 licences professionnelles et 5 spécialités de Master est une des réponses à ce défi.

Le projet d'ouverture de nouveaux parcours en formation à distance en est une autre.

Par ailleurs, l'université travaille à une refonte de l'information sur les diplômes au travers d'un nouveau portail WEB, qui tiendra compte, notamment, des besoins spécifiques des actifs et des entreprises.

Ce projet contribuera, d'une part, à l'élargissement à l'ensemble des licences professionnelles et masters, des démarches VAE qui se concentrent aujourd'hui sur un petit nombre de diplômes, d'autre part, à l'essor quantitatif de la VAE ainsi qu'à l'optimisation des coûts d'information du public.

\section{Bibliographie}

[1] LOI n 2002-73 du 17 janvier 2002 de modernisation sociale

[2] Décret no 2002-590 du 24 avril 2002 pris pour l'application du premier alinéa de l'article L. 613-3 et de l'article L. 613-4 du code de l'éducation et relatif à la validation des acquis de l'expérience par les établissements d'enseignement supérieur

[3] Note d'Information $n^{\circ} 09.16$ du Ministère de l'Enseignement Supérieur et de la Recherche " La validation des acquis dans l'enseignement supérieur en $2007 »$
[3] Revue Education Permanente. N$^{\circ}$ 158/mars 2004 « Les acquis de l'expérience. Première partie». Sous la direction de ASTIER P.

[4] Revue Education Permanente. $N^{\circ}$ 159/juin 2004 « Les acquis de l'expérience. Seconde partie ». Sous la direction de ASTIER Ph.

[5] Revue Education Permanente. N $N^{\circ}$ 153/2002-4 «L'accompagnement dans tous ses états ». Sous la direction de BOUTINET J.-P. et PINEAU G.

[6] Revue Actualité de la Formation Permanente $N^{\circ} 182$ janvier/février 2003 « la validation des acquis de l'expérience en pratique »

[7] BARBIER J.-M. (dir. Publication). « Savoirs théoriques et savoirs d'action ». PUF 1996. 